\title{
Wound repair: role of immune-epithelial interactions
}

\author{
G Leoni ${ }^{1}$, P-A Neumann ${ }^{2}$, R Sumagin ${ }^{3}$, TL Denning ${ }^{4}$ and A Nusrat ${ }^{5}$
}

The epithelium serves as a highly selective barrier at mucosal surfaces. Upon injury, epithelial wound closure is orchestrated by a series of events that emanate from the epithelium itself as well as by the temporal recruitment of immune cells into the wound bed. Epithelial cells adjoining the wound flatten out, migrate, and proliferate to rapidly cover denuded surfaces and re-establish mucosal homeostasis. This process is highly regulated by proteins and lipids, proresolving mediators such as Annexin A1 protein and resolvins released into the epithelial milieu by the epithelium itself and infiltrating innate immune cells including neutrophils and macrophages. Failure to achieve these finely tuned processes is observed in chronic inflammatory diseases that are associated with non-healing wounds. An improved understanding of mechanisms that mediate repair is important in the development of therapeutics aimed to promote mucosal wound repair.

\section{MUCOSAL WOUND REPAIR}

Epithelial barriers at mucosal surfaces prevent unrestricted exchange of molecules between the external environment and internal tissue compartments. Thus, the epithelium is uniquely positioned to mediate cross-talk between mucosal immune cells and complex external environmental agents including microbes and antigens. Epithelial damage with disruption of the mucosal barrier or wounds is seen in a number of pathologic states such as inflammatory diseases, ischemic events, and following mechanical injury. ${ }^{1}$ Thus, efficient repair of this critical barrier is important in re-establishing homeostasis and suppressing mucosal inflammation.

Mucosal wound repair is orchestrated by spatial and temporal network of mediators from different cell types that include the epithelium itself, resident and recruited inflammatory cells, endothelial cells, and mesenchymal cells. These biological mediators ensure repair of the epithelial barrier as well as coordinated resolution of inflammation with removal of damaged cells. Physiological wound healing is characterized by sequential, yet overlapping, stages of hemostasis, inflammation, proliferation, and remodeling. ${ }^{2}$ After mucosal injury, platelets are recruited to the site of injury with subsequent formation of a fibrin-rich clot that serves as a mechanical plug at the wound site in addition to secreting molecules that recruit inflammatory cells to the damaged mucosa. The inflammatory response initiated in the wounded tissue is associated with an early recruitment of neutrophils that secrete antimicrobial agents such as proteases, chemokines, and cytokines, which also control the subsequent recruitment of monocytes that differentiate into macrophages in the wound bed.

Wound repair has been extensively studied in other systems such as the skin where recruited leukocytes have been reported to stimulate proliferation and migration of keratinocytes that cover denuded surfaces. The fibrin clot is replaced by a vesseland collagen-rich granulation tissue in which fibroblasts differentiate into myofibroblasts that promote contraction and closure of the wound. The last phase of wound healing includes maturation and remodeling of the new tissue with increase in tissue strength that is mediated by turnover and remodeling of the extracellular matrix. Since the adult human skin does not completely regenerate, scar formation is the consequence of normal skin injury repair. A variety of different cell types, including macrophages, fibroblasts, and contractile myofibroblasts, participate in the proliferative phase of wound repair and play a critical role in regulating the size and quality of the scar. Insufficient or excessive healing responses such as

\footnotetext{
${ }^{1}$ Institute for Cardiovascular Prevention, Ludwig-Maximilians-University Munich, Munich, Germany. ${ }^{2}$ Department of General and Visceral Surgery, University Clinic of Muenster, Muenster, Germany. ${ }^{3}$ Department of Pathology, Feinberg School of Medicine, Northwestern University, Chicago, Illinois, USA. ${ }^{4}$ Institute for Biomedical Sciences, Center for Inflammation, Infection, and Immunity, Georgia State University Atlanta, Atlanta, Georgia, USA and ${ }^{5}$ Department of Pathology, University of Michigan, Ann Arbor, Michigan, USA. Correspondence: A Nusrat (anusrat@umich.edu)
}

Received 31 October 2014; accepted 28 May 2015; published online 15 July 2015. doi:10.1038/mi.2015.63 
observed in hypertrophic scars result in a failure of mucosal wound healing with poor clinical outcome. A number of parallels exist at mucosal surfaces. However, depending on the mucosa involved, there are differences in the extent and temporal recruitment of leukocytes, inflammatory as well as pro-repair mediators, and the extent of collagen deposition. For example, skin scars contain increased number of type I procollagen immunopositive cells and fibronectin content compared to oral mucosal wounds. ${ }^{3}$

In summary, wound healing is a complex process that is orchestrated by a number of cell types encompassing epithelial cells, leukocytes, and stromal cells. In this review, we focus on some of the key events that coordinate epithelial wound healing.

\section{EPITHELIAL WOUND HEALING}

Epithelial wound healing involves coordinated migration and proliferation of epithelial cells. Epithelial cells adjacent to the wound migrate as a sheet to cover denuded surfaces, which is also referred to as "epithelial restitution". ${ }^{4}$ Such epithelial sheet migration requires dynamic and coordinated remodeling of cell-cell and cell-matrix adhesions. Epithelial cells at the leading edge extrude filamentous-actin-rich protrusions that dynamically adhere to the matrix and mediate forward movement of the epithelial sheet. Epithelial transmembrane integrins in focal adhesive contacts include $\beta 1$ integrin that mediate bidirectional cross-talk between the matrix and intracellular signaling events to influence epithelial cytoskeletal restructuring and polarity required to guide epithelial movement and control cell proliferation. ${ }^{5,6}$ A number of small GTPases coordinate cellular events during wound closure. These include the Rho GTPase family member, Racl that controls cellular protrusions at the leading edge. ${ }^{7}$ Expression of constitutively active Rac1 influences epithelial movement and proliferation by targeting $\beta 1$ integrin in cellular protrusions and modulating actin dynamics. ${ }^{7,8}$ In addition, formyl peptide receptor-1 (FPR1) signaling activates Rac1 and Rho kinase to regulate intestinal epithelial cell (IEC) migration and wound repair. ${ }^{9,10}$ Epithelial-derived secreted proteins that include epidermal growth factor (EGF) and transforming growth factor- $\beta 1$ (TGF- $\beta 1$ ) coordinate repair of the epithelial barrier. ${ }^{11}$ However, a secreted Wnt antagonist (Dickkopf-1) Dkk-1 modifies the activation of another small GTPase, Cdc42 to influence direction orientation of migrating IECs. ${ }^{12}$

Although small mucosal wounds efficiently reseal by epithelial sheet migration and contraction of actin-myosin cytoskeleton, additional epithelial proliferation is needed for resealing of larger wounds. Proliferation of the epithelium increases the number of cells that populate the wound thereby contributing to mucosal wound closure. The intestinal epithelium covers one of the largest mucosal surfaces and is comprised of simple columnar epithelial cells that are highly dynamic as they are continuously renewed by pluripotent stem cells residing in crypt-like structures. ${ }^{13,14}$ The turnover of IECs provides an additional challenge to the maintenance of epithelial continuity after injury. ${ }^{15}$ Tissue regeneration requires proper organization of stem cells for efficient re-establishment of mucosal homeostasis. ${ }^{16}$ Our understanding of different stem cell populations that reside in the base of intestinal epithelial crypts has extensively grown and has opened therapeutic avenues in regenerative medicine. ${ }^{17}$ The in vitro expansion of intestinal organ cultures developed from adult stem cells, socalled organoids that can be used to restore damaged mucosal tissue have shown promising results. ${ }^{18}$ Mesenchymal stem cells secrete growth factors and cytokines, which recruit progenitor cells or endogenous stem cells to the injured site to mediate wound repair. Importantly, mesenchymal stem cells are multipotent and have been reported to differentiate into a number of cell types. ${ }^{19,20}$ Topically delivered adipose-derived stem cells exhibit an activated fibroblast phenotype, enhance macrophage recruitment, and increase granulation tissue formation in the rabbit ear wounds. ${ }^{21}$ Thus, epithelial barrier repair is orchestrated by sequential events coordinated by a number of cell types that ensure appropriate healing of wounds.

Epithelial cell migration in healing wounds is influenced by a fibrinolytic enzyme, plasmin. Plasmin is derived from activated plasminogen in the fibrin clot at the site of injury. The importance of plasminogen in wound re-epithelialization is demonstrated by the lack of wound repair in mice lacking the plasminogen gene. ${ }^{22}$ In addition to plasmin, matrix metalloproteinases (MMPs) cleave and degrade collagens in the matrix and basal lamina, thus promoting cell migration. As wound healing progresses, the provisional matrix is replaced by a newly formed matrix, rich in collagen synthesized by fibroblasts migrating into the wound. The synthesis of specific extracellular matrix molecules by fibroblasts in the wound is regulated by TGF- $\beta 1$ and other proteins such as interleukin- $1 \alpha$ (IL-1 $\alpha$ ) and IL-1 $\beta$, IL-4, and vascular endothelial growth factor (VEGF). Recently, IL-6, identified in epithelial cells, CD4 ${ }^{+}$ lymphocytes and monocytes, has been reported to be induced early after injury and control wound repair by enhancing epithelial cell proliferation. ${ }^{23}$

Within 10 days after injury, myofibroblasts derived from fibroblasts in the wound bed express smooth muscle actin and generate strong contractile forces that facilitate wound contraction and repair. In addition, angiogenesis influenced by a number of growth factors including fibroblast growth factor-2 (FGF-2) synthesized by macrophages and damaged endothelial cells promote wound repair.

A number of epithelial signaling events come into play to coordinate wound closure. We have previously observed that a membrane-associated, actin-binding protein, Annexin A2 (ANXA2), is upregulated in migrating IECs and enhances wound closure by controlling dynamics of $\beta 1$ integrin at the cell surface essential for the active turnover of cell-matrix associations, epithelial cell migration, and wound closure. ${ }^{24}$ Recent studies have highlighted a role of reactive oxygen species (ROS) signaling in coordinating mucosal wound repair. ${ }^{25-28}$ Intestinal microbiota such as lactobacilli promote FPR1 signaling and mucosal wound repair. ${ }^{29}$ Thus, FPRs function as pattern recognition receptors during mucosal reparative responses. ${ }^{25,29}$ The epithelial ROS is generated by the 
dihydronicotinamide-adenine dinucleotide phosphate oxidase complex 1 (NOX1) in IECs in response to FPR1 signaling to promote epithelial cell migration, proliferation, and ultimately mucosal wound closure. ${ }^{27}$ Mice lacking FPR1 exhibit delayed intestinal mucosal wound healing and recovery from acute colitis. $^{25,30}$

Although a role for oxygen in mediating wound healing has been well known for decades, the importance of cellular oxygen sensing in reparative mechanisms is a relatively new area. In the zebrafish model system, epithelial cells were reported to generate hydrogen peroxide gradients around wounds, which influenced leukocyte recruitment and repair. ${ }^{31}$ A well-known regulator of oxygen homeostasis, the hypoxiainducible factor- $1 \alpha$ (HIF- $1 \alpha$ ) also facilitates oxygen delivery to oxygen-deprived tissues (injured tissue). Activation of HIF-1 $\alpha$ has been reported to be involved in a plethora of biological events, such as angiogenesis cell growth, proliferation, and wound healing. ${ }^{32-34}$ HIF- $1 \alpha$ activation promotes synthesis of downstream target proteins such as VEGF, heme oxygenase-1, and $\mathrm{C}-\mathrm{X}-\mathrm{C}$ chemokine receptor type 4 (CXCR4), and the CXCR4-ligand stromal cell-derived factor-1 (SDF-1) that in turn facilitate the reparative response. HIF- $1 \alpha$ has also been reported to promote the synthesis of $\beta 1$ integrin and epithelial wound repair. ${ }^{33}$ In addition, HIF- $1 \alpha$ increases expression of an anti-adhesive protein, decay-accelerating factor (CD55) that serves as an apical anti-adhesive ligand by promoting the release of neutrophils from the epithelial surface. ${ }^{34}$ Thus, HIF$1 \alpha$ and CD55 function to ultimately reduce the epithelialassociated inflammation and mucosal wound repair. ${ }^{33}$ Recently, it has been shown that during colitis HIF- $1 \alpha$ is stabilized in mucosal wounds and a gaseous mediator hydrogen sulfide $\left(\mathrm{H}_{2} \mathrm{~S}\right)$ is important for HIF- $1 \alpha$ stabilization. ${ }^{35} \mathrm{H}_{2} \mathrm{~S}$ is generated and upregulated by macrophages at sites of mucosal damage where it serves to promote resolution of inflammation and wound healing. ${ }^{36}$

Studies in multiple organ systems have reported a plethora of autocrine and juxtacrine protein and lipid mediators that coordinate and control wound repair. A number of chemokines, defensins, and growth factors that influence mucosal epithelial wound repair include TGF- $\alpha$ and TGF- $\beta$, EGF, FGF, keratinocyte growth factor, insulin-like growth factor (IGF)-1 and IGF-2, hepatocyte growth factor, cytokines IL-1 $\beta$, IL-2, IL-6, IL-10, and a recent cytokine with pleiotropic actions, IL-22. ${ }^{37,38}$ In acute mucosal injury during colitis, IL-22 released by mucosal $\mathrm{CD} 4{ }^{+} \mathrm{T}$ cells and neutrophils serves as a paracrine signaling mediator to promote epithelial repair. ${ }^{39,40}$ Reparative mechanisms mediated by IL-22 occur through signal transducer and activator of transcription (STAT3), a pleiotropic transcription factor with important function in cytokine signaling in a variety of tissues. ${ }^{41}$

One of the central phenomena in tissue regeneration is the employment of signaling pathways that play pivotal roles in controlling development and homeostasis. Among others, $\beta$ catenin, a key mediator of canonical Wnt signaling in stem cells, not only controls physiologic epithelial cell turnover, but it also contributes to wound repair. ${ }^{2,16}$ A number of other intrinsic epithelial signaling mechanisms have been reported to control wound repair. Genetic targeting of Smad5, a protein reported to mediate bone morphogenetic protein signals in IECs, leads to impaired wound healing. ${ }^{42}$ Furthermore, Toll-like receptor (TLR)-4 signaling in IECs leads to the induction of cyclooxygenase-2 and production of prostaglandin E2, ${ }^{43}$ which promote restitution of damaged epithelia. ${ }^{44}$ Interestingly, IECs regulate function of dentritic cells and macrophages by secreting thymic stromal lymphopoietin and TGF- $\beta$. Incubation of monocyte-derived dentritic cells with supernatants of bacteria-stimulated epithelial cells renders dentritic cells noninflammatory since they release IL-10 and promote T-regulatory cells and Th2 responses. ${ }^{44}$

Matrix components that underlie the epithelium also contribute in coordinating mucosal wound repair. In the skin, the basement membrane protein collagen VII (COL7A1) secures the attachment of the epidermis to the dermis and controls wound repair by promoting re-epithelialization through organization of laminin-332 at the dermal-epidermal junction, and by regulating dermal fibroblast migration and cytokine production in the granulation tissue. These recent findings, which were validated in human wounds support an important role of COL7A1 in mediating epithelial wound repair. ${ }^{45}$

During restoration of denuded surfaces, directional migration of cells is pivotal for tissue regeneration. Such coordinated migration is achieved by specific gradients of chemoattractants. Migrating cells themselves can modify gradients of mediators that influence wound closure by modulating the release of chemoattractants such as leukotriene $\mathrm{B}_{4}, \mathrm{LTB}_{4}{ }^{46}$ Integrins, in concert with neutrophil-derived $\mathrm{LTB}_{4}$ and other chemoattractants, promote local neutrophil interaction while forming a tight wound seal. ${ }^{47,48}$ Zebrafish epithelial-like cells migrate along the body of the fish to reach the tip of the tail in response to a gradient of SDF-1; interestingly, in the absence of SDF-1 or its receptor (CXCR4) cells fail to achieve directional migration. ${ }^{49}$ Intravascular chemokine gradients and also formyl peptide signals generated from necrotic cells guide neutrophils toward the injury. ${ }^{50}$ In summary, wound healing initiated upon injury is a highly coordinated process that is controlled by a number of cell types and mediators in the epithelial milieu.

\section{SPATIAL AND TEMPORAL RECRUITMENT OF LEUKOCYTES INTO THE INJURED MUCOSA}

In response to mucosal injury and loss of the epithelial barrier, coordinated recruitment of leukocytes plays an important role in mediating host defense as well as in release of mediators that coordinate the ultimate resolution of inflammation and wound repair. Activation of leukocytes is followed by enhanced adhesive ability and shape-change that together promote their attachment to endothelial cells under hydrodynamic forces while perivascular cells such as macrophages regulate the exit of leukocytes from the vascular lumen. ${ }^{51}$ Below, we highlight some of the key inflammatory components that come into play during the early vs. late phases of wound closure as seen in Figure 1. 


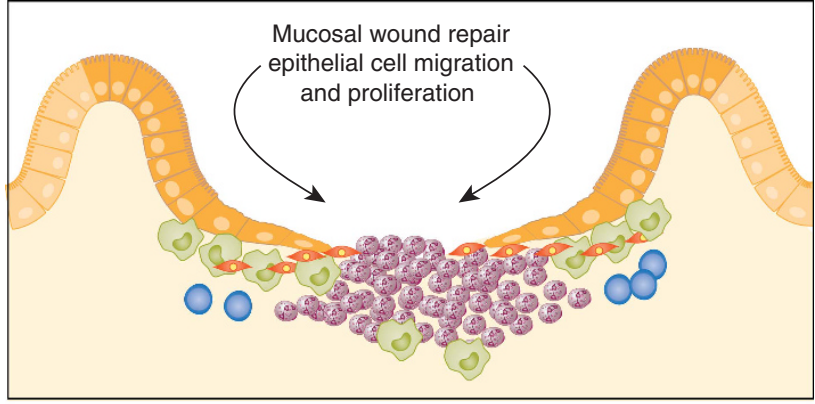

Epithelial cell

(6) Neutrophil Macrophage

Lymphocyte Fibroblast

Figure 1 Spatio-temporal control of wound closure. The initial phase of wound closure is characterized by an influx of neutrophils (day 1) followed by increased presence of macrophages (days 2-4) at the injured site. The inflammatory response is associated with re-epithelialization of the mucosa that is achieved by migration and subsequent proliferation of epithelial cells.

\section{EARLY PHASE OF WOUND HEALING}

Neutrophils are the first responders that arrive at the site of mucosal injury within the first $12 \mathrm{~h}$ and constitute nearly $50 \%$ of all cells at the wound site (Figure 2). ${ }^{52}$ Rapid mobilization of neutrophils from the circulation is primarily dependent on $\beta 2-$ integrin interactions with endothelial adhesion molecules, including intercellular adhesion molecule-1. ${ }^{53}$ Neutrophils have been considered to serve as a "double-edge sword" fulfilling both beneficial and detrimental roles in mucosal homeostasis. Antimicrobial activity of neutrophils is mediated by phagocytosis, release of ROS and lytic enzymes as well as formation of neutrophil extracellular traps that are composed of DNA, histones, and granular proteins such as neutrophil elastase. ${ }^{54}$ Neutrophil extracellular traps promote resolution of neutrophilic inflammation by degrading cytokines and chemokines in addition to reducing further neutrophil recruitment and activation. ${ }^{54}$

A recent study identified a role for the complement component $3 \mathrm{a}$ receptor $(\mathrm{C} 3 \mathrm{aR})$ in regulating neutrophil mobilization after acute intestinal injury, and highlighted C3aR antagonism as a potential treatment for neutrophildriven pathologies. ${ }^{55} \mathrm{C} 3 \mathrm{~b}$ covalently attaches to the surfaces of the cells in which complement is activated to target them for phagocytosis by macrophages. Interestingly, Sinno et al. ${ }^{56}$ also demonstrated that the topical application of C5 in a collagen vehicle also accelerated wound healing.

Neutrophils not only serve as scavengers to remove cellular debris but they also carry a vast arsenal of soluble mediators, including proteases and antimicrobial polypeptides that are essential for efficient recruitment of neutrophils toward the site of injury while also playing an important role in mediating physiologic epithelial repair. ${ }^{57}$ Neutrophils secrete anti-bacterial peptides referred to as defensins that further contribute in killing phagocytosed bacteria and facilitate wound healing in the skin ${ }^{58}$ and airway epithelium. ${ }^{59}$ Furthermore, neutrophils
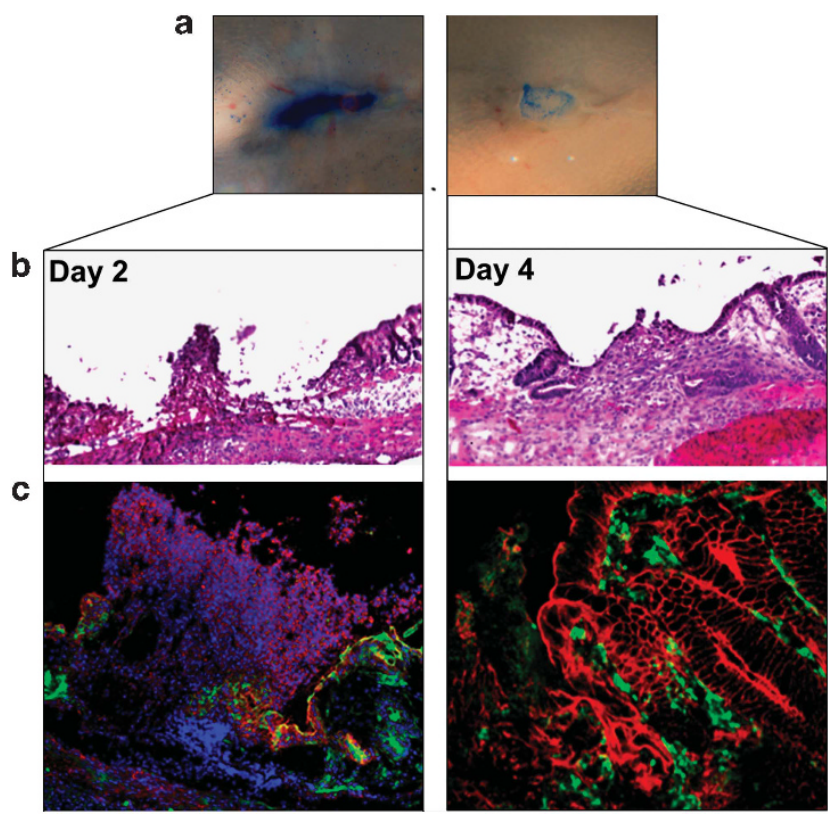

Figure 2 Resealing intestinal mucosal wounds. (a) Photographs obtained by colonoscopy of mice with resealing biopsy-induced colonic wounds on days 2 and 4 after injury highlighted with methylene blue. (b) Histology of intestinal mucosal wounds stained with hematoxylin and eosin. (c) Representative immunofluorescence confocal images showing neutrophils (visualized by anti-Ly6G staining, pink) and macrophages (visualized by anti-F4/80 staining, green) in intestinal mucosal wounds on days 2 and 4 post wounding.

secrete antimicrobial cationic peptides, LL-37 that has been shown to serve as a leukocyte chemoattractant ${ }^{60}$ in addition to stimulating airway epithelial cell proliferation and wound closure. $^{61}$ Mice genetically deficient for secretory leukocyte protease inhibitor display delayed cutaneous wound healing. The deficiency of secretory leukocyte protease inhibitor in these mice enhanced the inflammatory response and the elastase activity at the wound site, which ultimately led to delayed healing. ${ }^{62}$

Beneficial effects of neutrophils may be mediated by their direct interaction with epithelial ligands, or indirectly through synthesis and release of proresolving mediators. ${ }^{63,64}$ As such, neutrophil association with epithelia at mucosal surfaces activates $\beta$-catenin signaling to increase epithelial proliferation and promote wound closure. ${ }^{65}$ In addition, neutrophils are important sources of nitric oxide (NO) and TGF- $\beta$, both key regulators of wound healing; in fact, healing after acute intestinal epithelial injury is impaired in the presence of $\mathrm{NO}$ synthase inhibitors implicating $\mathrm{NO}$ an important mediator of mucosal wound closure. ${ }^{66}$ On the converse, neutrophils that traverse the epithelium influence cadherins in epithelial cells that mediate intercellular adhesion and neutrophil-derived proteases such as elastase have been reported to cleave the epithelial adherens junction cadherin, E-cadherin, thereby modifying epithelial adhesion that in turn influences epithelial wound closure. ${ }^{67}$

Neutrophils not only cleanse the wound of foreign particles, debris, and bacteria, but they also serve as an important source 
of proinflammatory cytokines. By day 3, the neutrophils are phagocytized either by macrophages or fibroblasts. Peripheral blood monocytes that continue to be recruited into the wound site differentiate into macrophages upon activation, further cleansing the wound and releasing cytokines and growth factors. Tauzin et al. recently reported a mechanism by which macrophages reduce inflammation by promoting neutrophil exit from the wounded tissue by a process referred to as reverse migration. Like neutrophils, macrophages are attracted to the injured site by ROS and Src family kinase signaling. Macrophages lacking the p22phox subunit of NOX2 or the tyrosine kinase Yrk were unable to migrate into wounds and promote the clearance of neutrophils in wounds. ${ }^{68}$

Defective neutrophil recruitment in individuals with neutrophil-specific granule deficiency and leukocyte adhesion deficiency results in frequent and repetitive infections leading to wound complications. On the converse, when in excess, neutrophils contribute to mucosal damage with impaired wound healing as observed in chronic inflammatory disorders such as inflammatory bowel disease. ${ }^{69,70}$ Infiltrating neutrophils in the mucosa trigger epithelial signaling events that control barrier properties and repair; ${ }^{71}$ however, the specific contribution of neutrophils on wound repair still remains elusive. A number of conflicting reports have argued both the detrimental and beneficial effects of neutrophils in mediating wound closure. Early studies in the 1970s showed that depletion of neutrophils with antisera in guinea pigs did not significantly influence healing of cutaneous wounds. ${ }^{72}$ More recent reports in the dental literature emphasize the role of macrophages and neutrophils in controlling tissue regeneration and repair. ${ }^{73}$ In gingival tissues, inflammatory mediators promote repair and dentin formation by odontoblasts and the differentiation of progenitor cells into a repair phenotype. If expression of these mediators persists, however, inflammation becomes sustained in the pulp, creating uncontrolled cell proliferation, angiogenesis, and collagen synthesis. ${ }^{3,74}$ On the converse, another study reported increased wound healing in the absence of neutrophils. ${ }^{75}$ These reports imply that at least under sterile conditions neutrophils are either not essential or perhaps detrimental for wound repair. In contrast, another study reported that neutrophil depletion in aging mice delayed wound repair supporting beneficial effects of neutrophils in wound closure. ${ }^{76}$ These differences could be related to extent of neutrophil depletion in the respective experimental models and the organ/model systems that were used to study wound repair. While in the circulation neutrophils are short lived, and it is now appreciated that following their migration across endothelia/epithelia their apoptosis is inhibited. ${ }^{77}$ As a result, neutrophils recruited to sites of mucosal injury may reside there for extended time periods. Thus, assays to evaluate neutrophil depletion in the circulation may not accurately reflect their loss in mucosal compartments, and therefore make the correlation of neutrophil loss and wound closure variable. These studies also suggest that a delicate balance of neutrophil numbers in the wound determines their ultimate influence on wound closure. Future studies to analyze and correlate extent of neutrophil loss and mucosal wound repair will therefore be helpful in determining their role in controlling wound repair.

In summary, a number of studies have implicated a crucial role of neutrophils in promoting wound healing and contributing to the resolution of inflammation after injury.

\section{LATE PHASE OF WOUND HEALING}

Like neutrophils, macrophages are an important component of the innate immune response to intestinal injury and wound repair, in part due to their ability to initiate and resolve inflammation and to communicate with other innate and adaptive immune cells. In general, macrophages can be classified into two major subtypes, which include inflammatory monocytes that rapidly differentiate into activated macrophages (M1 macrophages) and tissue-resident macrophages (M2 macrophages). ${ }^{78}$ M1 macrophages are phagocytes and along with neutrophils they are critical for mounting a protective immune response and in dealing with invading pathogens. M1 macrophages secrete proinflammatory cytokines such as tumor necrosis factor, IL-1, IL-6, and IL-12 as well as ROS. ${ }^{78-80}$ In contrast, tissue-resident macrophages have intrinsic anti-inflammatory functions, which play a key role in maintaining homeostasis and in promoting healing. Tissueresident macrophages antagonize the inflammatory responses of activated macrophages, thus promoting resolution of inflammation and allowing initiation of healing. Their effects occur, for example, through secretion of IL-10, a key antiinflammatory cytokine ${ }^{81}$ and novel proresolving lipid products such as maresins (MaRs), resolvins (Rvs), and protectins. MaRs exert potent proresolving and tissue homeostatic actions by inhibition of proinflammatory mediator production. ${ }^{82}$ In addition, macrophages produce neuroprotectin/protectin D1, which has been implicated in accelerating diabetic wound healing. ${ }^{83}$ Macrophages are also known to be a key source of $\mathrm{NO},{ }^{84}$ which is mainly used for pathogen killing. However, NO also plays an important role in the early phase of wound healing by contributing to re-epithelialization, collagen deposition, and wound repair. ${ }^{85}$ Although cues controlling the balance between macrophage subsets continue to emerge, recent evidence suggests that plasticity of phenotypes may also be involved. For example, during resolution of inflammation, activated M1 macrophages can acquire the phenotype of tissue-resident macrophages. ${ }^{86}$ Through their phagocytic activity, and secretion of immunomodulatory factors and cytokines, macrophages have been reported to contribute to bacterial clearance and wound debridement, collagen production, and wound contraction. ${ }^{78}$ The critical role of macrophages in wound healing was first described in 1970 s and $1980 \mathrm{~s}^{87}$ and subsequently confirmed in murine experimental models. Experiments in the 1970s established the concept that under sterile conditions, the influx of macrophages is essential for efficient healing of incisional skin wounds, whereas the influx of neutrophils was not critical. ${ }^{72,88}$ The importance of macrophages in mediating physiological wound repair has been elucidated in mice with attenuated number of macrophages at the wound site that exhibit delayed wound healing. ${ }^{89,90}$ Studies 
of repair in the skin with temporal and conditional depletion of macrophages have revealed that a reduction in macrophages during the inflammatory phase of wound closure impaired epithelialization, but reduced the formation of vascularized granulation tissue and scar formation. However, macrophage depletion in the subsequent repair phase resulted in severe hemorrhage and defects in wound closure. Finally, macrophage depletion during the late stage of repair (phase of tissue maturation) did not significantly impact the outcome of the repair response. These findings support a temporal role of macrophages in mucosal repair. ${ }^{91}$

Following mucosal damage, inflammatory monocytes are initially recruited into the mucosal wound site after the first wave of neutrophil infiltration. Monocytes recruited to sites of injury rapidly differentiate into so-called "wound-associated macrophages". ${ }^{92,93}$ Two to three days post injury, macrophages represent one of the predominant inflammatory cell components in the wound bed. ${ }^{94}$ In addition to their wellcharacterized anti-bacterial activities at the wound site, macrophages have also been reported to stimulate the intestinal epithelial progenitor niche during injury in order to help maintain the epithelial barrier. ${ }^{95,96}$ Macrophages further coordinate wound closure by secreting cytokines and growth factors that play a pivotal role in restructuring of the wound bed with accompanying matrix reorganization, angiogenesis, and epithelial barrier repair. ${ }^{97}$ One important cytokine in this regard is TGF- $\beta$. Remarkably, mice harboring a macrophagespecific deletion of the gene encoding $\beta$-catenin exhibited delayed skin wound healing associated with macrophagespecific defects in migration, adhesion to fibroblasts, and ability to produce TGF- $\beta 1$. Thus, $\beta$-catenin-mediated macrophage motility contributes to the scar size following cutaneous injury. ${ }^{98}$ In addition, colonic macrophages express the triggering receptor expressed on myeloid cells 2 (Trem2) and Trem2-null mice exhibit delayed wound healing. ${ }^{96}$ Furthermore, macrophages may be activated via TLRMyD88 pathway and contribute to the repair of intestinal mucosa by inducing regenerative responses by epithelial progenitors cells. ${ }^{95}$ However, uncontrolled release of proinflammatory and cytotoxic mediators by macrophages may also be detrimental to tissue repair in pathologic conditions. Indeed, increased numbers of macrophages during inflammation is a hallmark of an attenuated repair response in human diseases such as diabetes mellitus, vascular disease, and aging. ${ }^{88,99}$

Thus, macrophages are involved in many aspects of wound healing and their precise role depends on the ability to integrate cues from the microenvironment.

\section{MEDIATORS OF RESOLUTION OF INFLAMMATION AND WOUND HEALING}

Repair of wounds requires timely resolution of inflammation that is an active process. In this context, we have previously reported that ANXA1 protein (shown in Figure 3), expressed in epithelial cells and phagocytes, promotes IEC wound repair by activating FPRs (FPR1 and FPR2/ALX; FPR1 and FPR2 also called ALX -the lipoxin A4 receptor) and NOX1 signaling. ${ }^{27}$ In

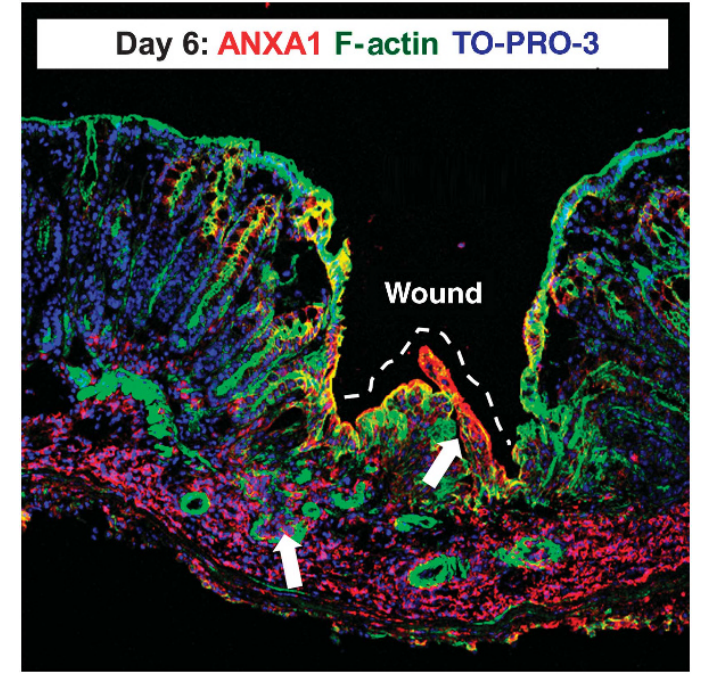

Figure 3 Mucosal ANXA1 expression is increased in repairing intestinal mucosal wounds. Representative image of murine colonic mucosa 2 days after biopsy-induced injury. Frozen sections were stained with antibodies against ANXA1 (red), F-actin (phalloidin, green), and nuclei (TO-PRO-3, blue). Arrows show ANXA1 expression by epithelial and immune cells in the injured mucosa.

addition, ANXA1-derived peptide Ac2-26 counteracts integrin activation and apoptosis of neutrophils, events involved in the resolution of inflammation and wound repair. ${ }^{100,101}$ Recently, we demonstrated that ANXA1 is released as a component of extracellular vesicles derived from IECs, where it activates wound repair circuits. Secreted ANXA1 containing extracellular vesicles were elevated in the serum of patients with active mucosal inflammation in inflammatory bowel disease. We further demonstrated that the local intestinal delivery of an exogenous ANXA1 mimetic peptide, Ac2-26 encapsulated in targeted polymeric nanoparticles, promotes mucosal wound repair and resolution of inflammation in a model of murine colitis. ${ }^{102}$ Another study highlighted the importance of these polymeric nanoparticles in the resolution of atherosclerosis. ${ }^{103}$ The anti-inflammatory and proresolving peptide chemerin 15, a ligand for the G-protein-coupled chemokine-like receptor-1 expressed on macrophages, neutrophils, and epithelial cells has also been reported to accelerate wound closure. ${ }^{104}$

In addition to protein and peptides, lipid mediators including eicosanoids and omega-3 derived "specialized pro-resolution mediators" (SPMs) such as lipoxins (LXs), Rvs, protectins, and MaRs control the acute inflammatory response by activating local resolution programs and tissue repair. ${ }^{105,106}$ SPMs generated in the resolution phase, limit leukocyte infiltration and enhance phagocytic activity of macrophages to remove apoptotic cells and/or microbes. Furthermore, SPMs stimulate the clearance of polymorphonuclear leukocytes from mucosal surfaces and contribute to their antimicrobial activity. $\mathrm{LXA}_{4}$ facilitates recovery of corneal epithelial wounds ${ }^{107,108}$ and recent studies have highlighted the importance of RvD1 in a model of acute zymosan-induced peritoneal inflammation by decreasing $\mathrm{LTB}_{4}$ biosynthesis and promoting $\mathrm{LXA}_{4}$ generation in macrophages. This effect of 
RvD1 is mediated by suppression of a calcium-sensing kinase calcium-calmodulin-dependent protein kinase II. ${ }^{109}$ Neutrophils in wounds shed microparticles containing ANXA1 that activate FPR2/ALX signaling in leukocytes, ${ }^{110}$ as well as in the epithelium to promote resolution of inflammation and repair. ${ }^{27}$ Furthermore, human neutrophil-derived microparticles enriched in RvD1 and $\mathrm{LXA}_{4}$ analogs have been reported to facilitate resolution of inflammation and repair. ${ }^{111}$ In humans, LXs are in large part biosynthesized by transcellular metabolic events, which occur when leukocytes interact with epithelial cells of the gastrointestinal tract or bronchial tissue within the vasculature. ${ }^{112}$ Recent studies have highlighted a role of the MaR family of mediators, such as MaR1 that are generated by immune cells during self-limited infections, and in the human milk to promote tissue regeneration and repair. ${ }^{113}$ Interestingly, structural elucidation of MaRs family members indicates that they are peptido-lipid mediators, namely sulfido-conjugate MaRs.

Another member of SMPs is RvD2 that influences leukocyte function and recruitment by promoting nitric oxide generation, and by modulating leukocyte adhesion receptor expression. ${ }^{114}$ Recent evidence indicates that aging mice display a dysregulated resolution response to acute inflammation, and the SPM RvD3 has beneficial effects in this scenario. ${ }^{115,116}$ Thus, SPMs have potential as next generation therapeutics for promoting resolution of inflammation and reparative responses without the immunosuppressive effects observed with current antiinflammatory drugs. ${ }^{112,114}$ SPMs indeed increase survival from bacterial and viral infections in mice. For example, RvE1 has been shown to have beneficial effects in a murine model of pneumonia by influencing proinflammatory cytokine secretion, neutrophil recruitment, and bacterial survival. ${ }^{117}$

In conclusion, endogenous proresolving proteins/peptides and lipid mediators released by a number of cell types orchestrate the resolution of inflammation and wound repair. Given these beneficial effects, proresolving mediators also represent ideal candidates that can be used as therapeutic agents to facilitate healing of wounds in chronic disorders.

\section{CHRONIC INFLAMMATION AND DELAYED WOUND HEALING}

Delayed or incomplete wound healing is observed in chronic inflammatory disorders such as diabetes, psoriasis, inflammatory bowel disease, systemic or local bacterial infections, chronic ischemia, or secondary to general immune suppression. Chronic wounds are defined as barrier defects that have not proceeded through timely repair events. In general there is an imbalance of pro- and anti-inflammatory stimuli at sites of injury in chronic diseases. Several factors, as presented and described in this review, including compromised immunological status and advanced age contribute to poor wound healing. Although inflammation is a pivotal component of the healing process, uncontrolled inflammation is detrimental to tissue regeneration. In contrast to early events in wound closure and early stages of inflammation, pathology of aberrant mucosal healing in chronic diseases is not well understood. ${ }^{118}$ Because of its known detrimental effect on wound closure a number of studies have examined chronic skin wound repair in patients with diabetes. ${ }^{119}$ Chronic wounds are characterized by an excessive inflammatory phase and the inability of the tissue to respond to reparative stimuli thereby leading to a chronically inflamed wound bed and failure to heal. The inflammatory response itself in chronic wounds differs from acute injury. ${ }^{120}$ Furthermore, chronic mucosal diseases with epithelial barrier disruption are associated with concomitant translocation of commensal bacteria that further perpetuate the inflammatory response (Figure 4). ${ }^{88}$ This in turn further damages the epithelium thereby contributing to the increased mucosal inflammation. Whereas during the acute phase neutrophils diminish after the first 24-72 h, in chronic wounds neutrophils continue to reside at sites of inflammation. ${ }^{121}$ One likely possibility for this phenomenon is the continued recruitment of neutrophils in response to invasion of bacteria at the injured site. ${ }^{122}$

Another major feature of non-healing wounds is the imbalance of extracellular protease activity such as MMPs in the inflammatory milieu. ${ }^{48,123}$ The failure of chronic wound healing has been attributed in part to deregulation of proteases and their inhibitors. ${ }^{124}$ Increased activity of MMPs such as collagenase and gelatinase A and B has been observed in chronic compared to acute wounds. ${ }^{125}$ Increased MMP activity influences epithelial adhesion and is also associated with degradation of growth factors and other peptides involved in the repair process, ${ }^{126}$ and ultimately results in immune-mediated tissue injury such as seen in inflammatory

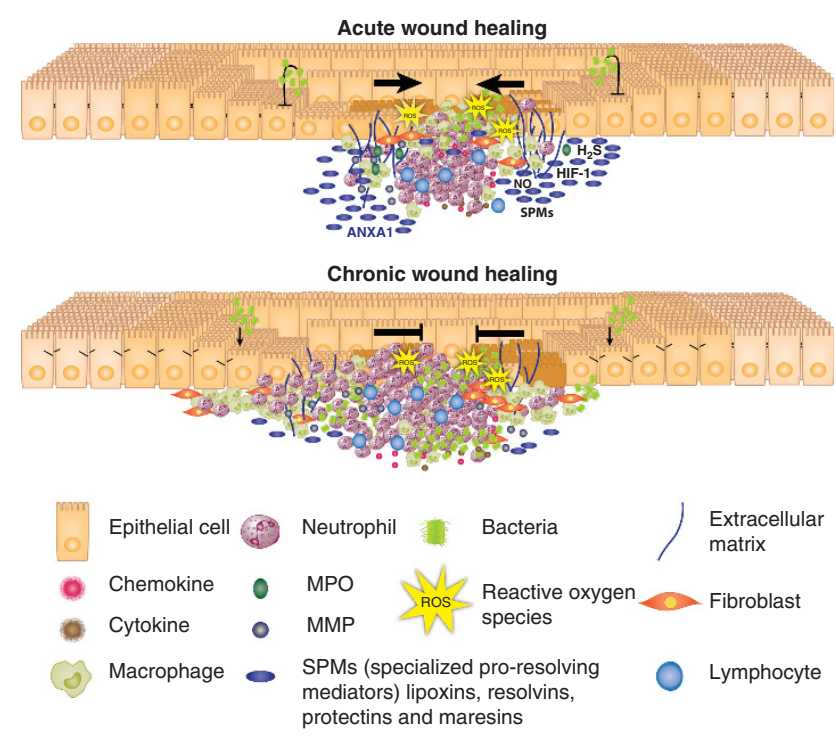

Figure 4 Acute and chronic wounds. During acute wound closure a plethora of signaling molecules emanating from the epithelium and immune cells coordinate wound repair (upper panel). In addition to cytokines, chemokines and growth factors, a distinct set of proresolving mediators such as protectins, resolvins, maresins, and proteins such as ANXA1 orchestrate wound repair. Chronic wounds (lower panel) are characterized by the presence of a high number of inflammatory cells (neutrophils) that generate a predominantly proinflammatory milieu, characterized by high concentrations of reactive oxygen species (ROS) as well as matrix metalloproteinases (MMPs) that in turn lead to destruction of the extracellular matrix, disruption of epithelial contacts, and result in further invasion of pathogens. HIF-1, hypoxia-inducible factor-1; MPO, myeloperoxidase. 
bowel disease. ${ }^{127-129}$ Furthermore, inflammatory mediators have been reported to influence migration of fibroblasts and epithelial cells and therefore impact the rate of wound closure in chronic intestinal inflammation. ${ }^{130,131}$ Altogether these events lead to instability of the extracellular matrix and prevent interactions between cells and matrix proteins that are essential for cell migration and wound resealing.

\section{CONCLUDING REMARKS}

The mucosal epithelium is at the interface of the external environment containing bacteria and antigens, and internal tissue compartments. Thus, repair of the mucosal surface does not occur in a sterile environment. Clinical and experimental studies have highlighted the importance of inflammation in coordinating mucosal wound repair. A delicate balance of proand anti-inflammatory mediators orchestrates the cross-talk of neutrophils, macrophages, and the epithelium to promote wound repair. An imbalance of the inflammatory milieu results in delayed wound repair seen in chronic inflammatory diseases. An improved understanding of pro-repair mediators is important for the design of therapeutic agents to promote healing of wounds in such chronic diseases.

\section{DISCLOSURE}

The authors declare no conflict of interest.

(c) 2015 Society for Mucosal Immunology

\section{REFERENCES}

1. Peterson, L.W. \& Artis, D. Intestinal epithelial cells: regulators of barrier function and immune homeostasis. Nat. Rev. Immunol. 14, 141-153 (2014).

2. Gurtner, G.C., Werner, S., Barrandon, Y. \& Longaker, M.T. Wound repair and regeneration. Nature 453, 314-321 (2008).

3. Wong, J.W. et al. Wound healing in oral mucosa results in reduced scar formation as compared with skin: evidence from the red Duroc pig model and humans. Wound Repair Regen. 17, 717-729 (2009).

4. Nusrat, A., Delp, C. \& Madara, J.L. Intestinal epithelial restitution. Characterization of a cell culture model and mapping of cytoskeletal elements in migrating cells. J. Clin. Invest. 89, 1501-1511 (1992).

5. Yang, H.W. et al. Cooperative activation of PI3K by Ras and Rho family small GTPases. Mol. Cell 47, 281-290 (2012).

6. Lotz, M.M., Nusrat, A., Madara, J.L., Ezzell, R., Wewer, U.M. \& Mercurio, A.M. Intestinal epithelial restitution. Involvement of specific laminin isoforms and integrin laminin receptors in wound closure of a transformed model epithelium. Am. J. Pathol. 150, 747-760 (1997).

7. Yamaguchi, N., Mizutani, T., Kawabata, K. \& Haga, H. Leader cells regulate collective cell migration via Rac activation in the downstream signaling of integrin beta1 and PI3K. Sci. Rep. 5, 7656 (2015).

8. Ray, R.M., McCormack, S.A., Covington, C., Viar, M.J., Zheng, Y. \& Johnson, L.R. The requirement for polyamines for intestinal epithelial cell migration is mediated through Rac1. J. Biol. Chem. 278, 13039-13046 (2003).

9. Babbin, B.A. et al. Formyl peptide receptor-1 activation enhances intestinal epithelial cell restitution through phosphatidylinositol 3-kinasedependent activation of Rac1 and Cdc42. J. Immunol. 179, 8112-8121 (2007).

10. Hopkins, A.M., Pineda, A.A., Winfree, L.M., Brown, G.T., Laukoetter, M.G. \& Nusrat, A. Organized migration of epithelial cells requires control of adhesion and protrusion through Rho kinase effectors. Am. J. Physiol. Gastrointest. Liver Physiol. 292, G806-G817 (2007).

11. Leydon, C., Imaizumi, M., Bartlett, R.S., Wang, S.F. \& Thibeault, S.L. Epithelial cells are active participants in vocal fold wound healing: an in vivo animal model of injury. PLoS One 9, e115389 (2014).
12. Koch, S. et al. Dkk-1 inhibits intestinal epithelial cell migration by attenuating directional polarization of leading edge cells. Mol. Biol. Cell 20, 4816-4825 (2009).

13. Crosnier, C., Stamataki, D. \& Lewis, J. Organizing cell renewal in the intestine: stem cells, signals and combinatorial control. Nat. Rev. Genet. 7, 349-359 (2006).

14. van der Flier, L.G. \& Clevers, H. Stem cells, self-renewal, and differentiation in the intestinal epithelium. Annu. Rev. Physiol. 71, 241-260 (2009).

15. Eisenhoffer, G.T. et al. Crowding induces live cell extrusion to maintain homeostatic cell numbers in epithelia. Nature 484, 546-549 (2012).

16. Miyoshi, H., Ajima, R., Luo, C.T., Yamaguchi, T.P. \& Stappenbeck, T.S. Wnt5a potentiates TGF-beta signaling to promote colonic crypt regeneration after tissue injury. Science 338, 108-113 (2012).

17. Shaker, A. \& Rubin, D.C. Stem cells: one step closer to gut repair. Nature 485, 181-182 (2012).

18. Sato, T. et al. Single Lgr5 stem cells build crypt-villus structures in vitro without a mesenchymal niche. Nature 459, 262-265 (2009).

19. Phinney, D.G. \& Prockop, D.J. Concise review: mesenchymal stem/ multipotent stromal cells: the state of transdifferentiation and modes of tissue repair-current views. Stem Cells 25, 2896-2902 (2007).

20. Stappenbeck, T.S. \& Miyoshi, H. The role of stromal stem cells in tissue regeneration and wound repair. Science 324, 1666-1669 (2009).

21. Hong, S.J. et al. Topically delivered adipose derived stem cells show an activated-fibroblast phenotype and enhance granulation tissue formation in skin wounds. PLoS One 8, e55640 (2013).

22. Green, K.A. et al. Profibrinolytic effects of metalloproteinases during skin wound healing in the absence of plasminogen. J. Invest. Dermatol. 128, 2092-2101 (2008).

23. Kuhn, K.A., Manieri, N.A., Liu, T.C. \& Stappenbeck, T.S. IL-6 stimulates intestinal epithelial proliferation and repair after injury. PLoS One 9, e114195 (2014).

24. Rankin, C.R. et al. Annexin A2 regulates beta1 integrin internalization and intestinal epithelial cell migration. J. Biol. Chem. 288, 15229-15239 (2013).

25. Alam, A. et al. Redox signaling regulates commensal-mediated mucosal homeostasis and restitution and requires formyl peptide receptor 1. Mucosal Immunol. 7, 645-655 (2014).

26. Swanson, P.A. II et al. Enteric commensal bacteria potentiate epithelial restitution via reactive oxygen species-mediated inactivation of focal adhesion kinase phosphatases. Proc. Natl. Acad. Sci. USA 108, 88038808 (2011).

27. Leoni, G. et al. Annexin A1, formyl peptide receptor, and NOX1 orchestrate epithelial repair. J. Clin. Invest. 123, 443-454 (2013).

28. Hunt, T.K., Zederfeldt, B. \& Goldstick, T.K. Oxygen and healing. Am. J. Surg. 118, 521-525 (1969).

29. Wentworth, C.C., Alam, A., Jones, R.M., Nusrat, A. \& Neish, A.S. Enteric commensal bacteria induce extracellular signal-regulated kinase pathway signaling via formyl peptide receptor-dependent redox modulation of dual specific phosphatase 3. J. Biol. Chem. 286, 38448-38455 (2011).

30. Chen, K. et al. Formylpeptide receptor-2 contributes to colonic epithelial homeostasis, inflammation, and tumorigenesis. J. Clin. Invest. 123, 1694-1704 (2013).

31. Niethammer, P., Grabher, C., Look, A.T. \& Mitchison, T.J. A tissue-scale gradient of hydrogen peroxide mediates rapid wound detection in zebrafish. Nature 459, 996-999 (2009).

32. Ahn, G.O. et al. Transcriptional activation of hypoxia-inducible factor-1 (HIF-1) in myeloid cells promotes angiogenesis through VEGF and S100A8. Proc. Natl. Acad. Sci. USA 111, 2698-2703 (2014).

33. Campbell, E.L. et al. Transmigrating neutrophils shape the mucosal microenvironment through localized oxygen depletion to influence resolution of inflammation. Immunity 40, 66-77 (2014).

34. Louis, N.A., Hamilton, K.E., Kong, T. \& Colgan, S.P. HIF-dependent induction of apical CD55 coordinates epithelial clearance of neutrophils. FASEB J. 19, 950-959 (2005).

35. Flannigan, K.L. et al. Proresolution effects of hydrogen sulfide during colitis are mediated through hypoxia-inducible factor-1alpha. FASEB $\mathrm{J}$. 29, 1531-1602 (2014).

36. Wallace, J.L., Ferraz, J.G. \& Muscara, M.N. Hydrogen sulfide: an endogenous mediator of resolution of inflammation and injury. Antioxid. Redox Signal. 17, 58-67 (2012). 
37. Werner, S. \& Grose, R. Regulation of wound healing by growth factors and cytokines. Physiol. Rev. 83, 835-870 (2003).

38. Beck, P.L., Rosenberg, I.M., Xavier, R.J., Koh, T., Wong, J.F. \& Podolsky, D.K. Transforming growth factor-beta mediates intestinal healing and susceptibility to injury in vitro and in vivo through epithelial cells. Am. J. Pathol. 162, 597-608 (2003).

39. Zindl, C.L. et al. IL-22-producing neutrophils contribute to antimicrobial defense and restitution of colonic epithelial integrity during colitis. Proc. Natl. Acad. Sci. USA 110, 12768-12773 (2013).

40. Denning, T.L. \& Parkos, C.A. Neutrophils enlist IL-22 to restore order in the gut. Proc. Natl. Acad. Sci. USA 110, 12509-12510 (2013).

41. Pickert, G. et al. STAT3 links IL-22 signaling in intestinal epithelial cells to mucosal wound healing. J. Exp. Med. 206, 1465-1472 (2009).

42. Grinnell, F., Ho, C.H. \& Wysocki, A. Degradation of fibronectin and vitronectin in chronic wound fluid: analysis by cell blotting, immunoblotting, and cell adhesion assays. J. Invest. Dermatol. 98, 410-416 (1992).

43. Fukata, M. et al. Cox-2 is regulated by Toll-like receptor-4 (TLR4) signaling: role in proliferation and apoptosis in the intestine. Gastroenterology 131, 862-877 (2006).

44. Morteau, O. et al. Impaired mucosal defense to acute colonic injury in mice lacking cyclooxygenase-1 or cyclooxygenase-2. J. Clin. Invest. 105, 469-478 (2000).

45. Castaneda, C.A. \& Gomez, H.L. Targeted therapies: combined lapatinib and paclitaxel in HER2-positive breast cancer. Nat. Rev. Clin. Oncol. 6, 308-309 (2009).

46. Martin, P. et al. Wound healing in the PU. 1 null mouse-tissue repair is not dependent on inflammatory cells. Curr. Biol. 13, 1122-1128 (2003).

47. Lammermann, T. et al. Neutrophil swarms require LTB4 and integrins at sites of cell death in vivo. Nature 498, 371-375 (2013).

48. Eming, S.A., Krieg, T. \& Davidson, J.M. Inflammation in wound repair: molecular and cellular mechanisms. J. Invest. Dermatol. 127, 514-525 (2007).

49. Demidova-Rice, T.N., Hamblin, M.R. \& Herman, I.M. Acute and impaired wound healing: pathophysiology and current methods for drug delivery, part 2: role of growth factors in normal and pathological wound healing: therapeutic potential and methods of delivery. Adv. Skin Wound Care 25, 349-370 (2012).

50. Rutter, M.D. et al. Thirty-year analysis of a colonoscopic surveillance program for neoplasia in ulcerative colitis. Gastroenterology 130, 10301038 (2006).

51. Nourshargh, S. \& Alon, R. Leukocyte migration into inflamed tissues. Immunity 41, 694-707 (2014).

52. Engelhardt, E., Toksoy, A., Goebeler, M., Debus, S., Brocker, E.B. \& Gillitzer, R. Chemokines IL-8, GROalpha, MCP-1, IP-10, and Mig are sequentially and differentially expressed during phase-specific infiltration of leukocyte subsets in human wound healing. Am. J. Pathol. 153, 18491860 (1998).

53. Sumagin, R. \& Sarelius, I.H. Intercellular adhesion molecule-1 enrichment near tricellular endothelial junctions is preferentially associated with leukocyte transmigration and signals for reorganization of these junctions to accommodate leukocyte passage. J. Immunol. 184, 5242-5252 (2010).

54. Schauer, C. et al. Aggregated neutrophil extracellular traps limit inflammation by degrading cytokines and chemokines. Nat. Med. 20, 511-517 (2014).

55. Wu, M.C. et al. The receptor for complement component C3a mediates protection from intestinal ischemia-reperfusion injuries by inhibiting neutrophil mobilization. Proc. Natl. Acad. Sci. USA 110, 9439-9444 (2013).

56. Sinno, H. et al. Accelerated wound healing with topical application of complement C5. Plast. Reconstr. Surg. 130, 523-529 (2012).

57. Nauseef, W.M. \& Borregaard, N. Neutrophils at work. Nat. Immunol. 15, 602-611 (2014).

58. Hirsch, T. et al. Human beta-defensin-3 promotes wound healing in infected diabetic wounds. J. Gene Med. 11, 220-228 (2009).

59. Aarbiou, J. et al. Neutrophil defensins enhance lung epithelial wound closure and mucin gene expression in vitro. Am. J. Respir. Cell Mol. Biol. 30, 193-201 (2004).

60. De, Y. et al. LL-37, the neutrophil granule- and epithelial cell-derived cathelicidin, utilizes formyl peptide receptor-like 1 (FPRL1) as a receptor to chemoattract human peripheral blood neutrophils, monocytes, and T cells. J. Exp. Med. 192, 1069-1074 (2000).

61. Shaykhiev, R. et al. Human endogenous antibiotic LL-37 stimulates airway epithelial cell proliferation and wound closure. Am. J. Physiol. Lung Cell. Mol. Physiol. 289, L842-L848 (2005).

62. Ashcroft, G.S. et al. Secretory leukocyte protease inhibitor mediates nonredundant functions necessary for normal wound healing. Nat. Med. 6 , 1147-1153 (2000).

63. Serhan, C.N., Chiang, N. \& Van Dyke, T.E. Resolving inflammation: dual anti-inflammatory and pro-resolution lipid mediators. Nat. Rev. Immunol. 8, 349-361 (2008).

64. Spite, M. \& Serhan, C.N. Novel lipid mediators promote resolution of acute inflammation: impact of aspirin and statins. Circ. Res. 107, 1170-1184 (2010).

65. Zemans, R.L. et al. Neutrophil transmigration triggers repair of the lung epithelium via beta-catenin signaling. Proc. Natl. Acad. Sci. USA 108, 15990-15995 (2011).

66. Aoi, Y., Terashima, S., Ogura, M., Nishio, H., Kato, S. \& Takeuchi, K. Roles of nitric oxide (NO) and NO synthases in healing of dextran sulfate sodiuminduced rat colitis. J. Physiol. Pharmacol. 59, 315-336 (2008).

67. Ginzberg, H.H. et al. Neutrophil-mediated epithelial injury during transmigration: role of elastase. Am. J. Physiol. Gastrointest. Liver Physiol. 281, G705-G717 (2001).

68. Tauzin, S., Starnes, T.W., Becker, F.B., Lam, P.Y. \& Huttenlocher, A. Redox and Src family kinase signaling control leukocyte wound attraction and neutrophil reverse migration. J. Cell Biol. 207, 589-598 (2014).

69. Xavier, R.J. \& Podolsky, D.K. Unravelling the pathogenesis of inflammatory bowel disease. Nature 448, 427-434 (2007).

70. Fournier, B.M. \& Parkos, C.A. The role of neutrophils during intestinal inflammation. Mucosal Immunol. 5, 354-366 (2012).

71. Sumagin, R., Robin, A.Z., Nusrat, A. \& Parkos, C.A. Transmigrated neutrophils in the intestinal lumen engage ICAM-1 to regulate the epithelial barrier and neutrophil recruitment. Mucosal Immunol. 7, 905-915 (2014)

72. Simpson, D.M. \& Ross, R. The neutrophilic leukocyte in wound repair a study with antineutrophil serum. J. Clin. Invest. 51, 2009-2023 (1972).

73. Souza, C.M. et al. Regeneration of skin tissue promoted by mesenchymal stem cells seeded in nanostructured membrane. Transplant. Proc. 46, 1882-1886 (2014).

74. Smith, P.C., Caceres, M., Martinez, C., Oyarzun, A. \& Martinez, J. Gingival wound healing: an essential response disturbed by aging? J. Dent. Res. 94, 395-402 (2014).

75. Dovi, J.V., He, L.K. \& DiPietro, L.A. Accelerated wound closure in neutrophil-depleted mice. J. Leukoc. Biol. 73, 448-455 (2003).

76. Nishio, N., Okawa, Y., Sakurai, H. \& Isobe, K. Neutrophil depletion delays wound repair in aged mice. Age (Dordr) 30, 11-19 (2008).

77. Le'Negrate, G., Rostagno, P., Auberger, P., Rossi, B. \& Hofman, P. Downregulation of caspases and Fas ligand expression, and increased lifespan of neutrophils after transmigration across intestinal epithelium. Cell Death Differ. 10, 153-162 (2003).

78. Murray, P.J. \& Wynn, T.A. Protective and pathogenic functions of macrophage subsets. Nat. Rev. Immunol. 11, 723-737 (2011).

79. Sindrilaru, A. et al. An unrestrained proinflammatory M1 macrophage population induced by iron impairs wound healing in humans and mice. J. Clin. Invest. 121, 985-997 (2011).

80. Murray, P.J. et al. Macrophage activation and polarization: nomenclature and experimental guidelines. Immunity 41, 14-20 (2014).

81. Murray, P.J. The primary mechanism of the IL-10-regulated antiinflammatory response is to selectively inhibit transcription. Proc. Natl. Acad. Sci. USA 102, 8686-8691 (2005).

82. Dalli, J. et al. The novel 13S,14S-epoxy-maresin is converted by human macrophages to maresin 1 (MaR1), inhibits leukotriene A4 hydrolase (LTA4H), and shifts macrophage phenotype. FASEB J. 27, 2573-2583 (2013).

83. Hong, S. et al. Neuroprotectin/protectin D1: endogenous biosynthesis and actions on diabetic macrophages in promoting wound healing and innervation impaired by diabetes. Am. J. Physiol. Cell Physiol. 307, C1058-C1067 (2014).

84. Schaffer, M.R., Tantry, U., van Wesep, R.A. \& Barbul, A. Nitric oxide metabolism in wounds. J. Surg. Res. 71, 25-31 (1997). 
85. Stallmeyer, B., Kampfer, H., Kolb, N., Pfeilschifter, J. \& Frank, S. The function of nitric oxide in wound repair: inhibition of inducible nitric oxidesynthase severely impairs wound reepithelialization. J. Invest. Dermatol. 113, 1090-1098 (1999).

86. Xiao, W., Hong, H., Kawakami, Y., Lowell, C.A. \& Kawakami, T. Regulation of myeloproliferation and M2 macrophage programming in mice by Lyn/ Hck, SHIP, and Stat5. J. Clin. Invest. 118, 924-934 (2008).

87. Diegelmann, R.F., Cohen, I.K. \& Kaplan, A.M. The role of macrophages in wound repair: a review. Plast. Reconstr. Surg. 68, 107-113 (1981).

88. Neurath, M.F. \& Travis, S.P. Mucosal healing in inflammatory bowel diseases: a systematic review. Gut 61, 1619-1635 (2012).

89. Nagaoka, T. et al. Delayed wound healing in the absence of intercellular adhesion molecule-1 or L-selectin expression. Am. J. Pathol. 157, 237-247 (2000).

90. Ishida, Y., Gao, J.L. \& Murphy, P.M. Chemokine receptor CX3CR1 mediates skin wound healing by promoting macrophage and fibroblast accumulation and function. J. Immunol. 180, 569-579 (2008).

91. Bettenworth, D. et al. The tripeptide KdPT protects from intestinal inflammation and maintains intestinal barrier function. Am. J. Pathol. 179, 1230-1242 (2011).

92. Crane, M.J., Daley, J.M., van Houtte, O., Brancato, S.K., Henry, W.L. Jr \& Albina, J.E. The monocyte to macrophage transition in the murine sterile wound. PLoS One 9, e86660 (2014).

93. Wynn, T.A., Chawla, A. \& Pollard, J.W. Macrophage biology in development, homeostasis and disease. Nature 496, 445-455 (2013).

94. Witte, M.B. \& Barbul, A. General principles of wound healing. Surg. Clin. North Am. 77, 509-528 (1997).

95. Pull, S.L., Doherty, J.M., Mills, J.C., Gordon, J.I. \& Stappenbeck, T.S. Activated macrophages are an adaptive element of the colonic epithelial progenitor niche necessary for regenerative responses to injury. Proc. Natl. Acad. Sci. USA 102, 99-104 (2005).

96. Seno, H., Miyoshi, H., Brown, S.L., Geske, M.J., Colonna, M. \& Stappenbeck, T.S. Efficient colonic mucosal wound repair requires Trem2 signaling. Proc. Natl. Acad. Sci. USA 106, 256-261 (2009).

97. Lucas, T. et al. Differential roles of macrophages in diverse phases of skin repair. J. Immunol. 184, 3964-3977 (2010).

98. Amini-Nik, S. et al. beta-Catenin-regulated myeloid cell adhesion and migration determine wound healing. J. Clin. Invest. 124, 2599-2610 (2014).

99. Neurath, M.F. New targets for mucosal healing and therapy in inflammatory bowel diseases. Mucosal Immunol. 7, 6-19 (2014).

100. Dalli, J. et al. Proresolving and tissue-protective actions of annexin A1based cleavage-resistant peptides are mediated by formyl peptide receptor 2/lipoxin A4 receptor. J. Immunol. 190, 6478-6487 (2013).

101. Drechsler, M. et al. Annexin A1 counteracts chemokine-induced arterial myeloid cell recruitment. Circ. Res. 116, 827-835 (2015).

102. Leoni, G. et al. Annexin A1-containing extracellular vesicles and polymeric nanoparticles promote epithelial wound repair. J. Clin. Invest. 125, 1215-1227 (2015).

103. Fredman, G. et al. Targeted nanoparticles containing the proresolving peptide Ac2-26 protect against advanced atherosclerosis in hypercholesterolemic mice. Sci. Transl. Med. 7, 275ra20 (2015).

104. Cash, J.L., Bena, S., Headland, S.E., McArthur, S., Brancaleone, V. \& Perretti, M. Chemerin15 inhibits neutrophil-mediated vascular inflammation and myocardial ischemia-reperfusion injury through ChemR23. EMBO Rep. 14, 999-1007 (2013).

105. Serhan, C.N. Pro-resolving lipid mediators are leads for resolution physiology. Nature 510, 92-101 (2014)

106. Buckley, C.D., Gilroy, D.W. \& Serhan, C.N. Proresolving lipid mediators and mechanisms in the resolution of acute inflammation. Immunity 40 , 315-327 (2014).

107. Gronert, K. Lipoxins in the eye and their role in wound healing. Prostaglandins Leukot. Essent. Fatty Acids 2005)73, 221-229.

108. Gronert, K., Maheshwari, N., Khan, N., Hassan, I.R., Dunn, M. \& Laniado Schwartzman, M. A role for the mouse 12/15-lipoxygenase pathway in promoting epithelial wound healing and host defense. J. Biol. Chem. 280 15267-15278 (2005)

109. Fredman, G. et al. Resolvin D1 limits 5-lipoxygenase nuclear localization and leukotriene B4 synthesis by inhibiting a calcium- activated kinase pathway. Proc. Natl. Acad. Sci. USA 111, 14530 14535 (2014)

110. Dalli, J., Norling, L.V., Renshaw, D., Cooper, D., Leung, K.Y. \& Perretti, M. Annexin 1 mediates the rapid anti-inflammatory effects of neutrophilderived microparticles. Blood 112, 2512-2519 (2008).

111. Norling, L.V., Spite, M., Yang, R., Flower, R.J., Perretti, M. \& Serhan, C.N. Cutting edge: humanized nano-proresolving medicines mimic inflammation-resolution and enhance wound healing. J. Immunol. 186, 5543-5547 (2011).

112. Serhan, C.N. Resolution phase of inflammation: novel endogenous antiinflammatory and proresolving lipid mediators and pathways. Annu. Rev. Immunol. 25, 101-137 (2007).

113. Dalli, J., Chiang, N. \& Serhan, C.N. Identification of 14-series sulfidoconjugated mediators that promote resolution of infection and organ protection. Proc. Natl. Acad. Sci. USA 111, E4753-E4761 (2014).

114. Spite, M. et al. Resolvin D2 is a potent regulator of leukocytes and controls microbial sepsis. Nature 461, 1287-1291 (2009).

115. Sousounis, K., Baddour, J.A. \& Tsonis, P.A. Aging and regeneration in vertebrates. Curr. Top. Dev. Biol. 108, 217-246 (2014).

116. Arnardottir, H.H., Dalli, J., Colas, R.A., Shinohara, M. \& Serhan, C.N. Aging delays resolution of acute inflammation in mice: reprogramming the host response with novel nano-proresolving medicines. J. Immunol. 193, 4235-4244 (2014).

117. Seki, H. et al. The anti-inflammatory and proresolving mediator resolvin E1 protects mice from bacterial pneumonia and acute lung injury. J. Immunol. 184, 836-843 (2010).

118. Rieder, F. et al. Results of the 2nd scientific workshop of the ECCO (III): basic mechanisms of intestinal healing. J. Crohns Colitis 6, 373-385 (2012).

119. Demidova-Rice, T.N., Hamblin, M.R. \& Herman, I.M. Acute and impaired wound healing: pathophysiology and current methods for drug delivery, part 1: normal and chronic wounds: biology, causes, and approaches to care. Adv. Skin Wound Care 25, 304-314 (2012).

120. Rieder, F., Brenmoehl, J., Leeb, S., Scholmerich, J. \& Rogler, G. Wound healing and fibrosis in intestinal disease. Gut 56, 130-139 (2007).

121. Diegelmann, R.F. Excessive neutrophils characterize chronic pressure ulcers. Wound Repair Regen. 11, 490-495 (2003).

122. Martin, J.M., Zenilman, J.M. \& Lazarus, G.S. Molecular microbiology: new dimensions for cutaneous biology and wound healing. J. Invest. Dermatol. 130, 38-48 (2010).

123. Wysocki, A.B., Staiano-Coico, L. \& Grinnell, F. Wound fluid from chronic leg ulcers contains elevated levels of metalloproteinases MMP-2 and MMP-9. J. Invest. Dermatol. 101, 64-68 (1993).

124. Eming, S.A., Martin, P. \& Tomic-Canic, M. Wound repair and regeneration: mechanisms, signaling, and translation. Sci. Transl. Med. 6, 265sr6 (2014).

125. Yager, D.R., Zhang, L.Y., Liang, H.X., Diegelmann, R.F. \& Cohen, I.K. Wound fluids from human pressure ulcers contain elevated matrix metalloproteinase levels and activity compared to surgical wound fluids. J. Invest. Dermatol. 107, 743-748 (1996).

126. Lauer, G. et al. Expression and proteolysis of vascular endothelial growth factor is increased in chronic wounds. J. Invest. Dermatol. 115, 12-18 (2000).

127. von Lampe, B., Barthel, B., Coupland, S.E., Riecken, E.O. \& Rosewicz, S. Differential expression of matrix metalloproteinases and their tissue inhibitors in colon mucosa of patients with inflammatory bowel disease. Gut 47, 63-73 (2000).

128. Castaneda, F.E. et al. Targeted deletion of metalloproteinase 9 attenuates experimental colitis in mice: central role of epithelial-derived MMP. Gastroenterology 129, 1991-2008 (2005).

129. Nava, P., Kamekura, R. \& Nusrat, A. Cleavage of transmembrane junction proteins and their role in regulating epithelial homeostasis. Tissue Barriers 1, e24783 (2013).

130. Leeb, S.N. et al. Reduced migration of fibroblasts in inflammatory bowel disease: role of inflammatory mediators and focal adhesion kinase. Gastroenterology 125, 1341-1354 (2003).

131. Tong, Q. et al. Interferon-gamma inhibits T84 epithelial cell migration by redirecting transcytosis of beta1 integrin from the migrating leading edge. J. Immunol. 175, 4030-4038 (2005). 